\title{
Efficacy of Immunotherapy in Advanced Renal Cell Carcinoma (RCC) Patients Despite the Early Treatment Interruption Due to Serious Immune- related Adverse Events. Meet-Uro 13 Trial on Behalf of the MeetUro Investigators
}

Marco Stellato ( $\square$ m.stellato@unicampus.it)

Department of Medical Oncology, Campus Bio-Medico University of Rome, Rome https://orcid.org/0000-0002-0993-7540

\section{Giuseppe Procopio}

Medical Oncology Department, Fondazione IRCCS Istituto Nazionale dei Tumori, Milano

Ugo De Giorgi

Irstea: Institut National de Recherche pour l'Agriculture l'Alimentation et l'Environnement

\section{Marco Maruzzo}

Medical Oncology Unit 1, Department of Oncology, Istituto Oncologico Veneto IOV IRCCS, Padua

Davide Bimbatti

Medical Oncology Unit, Department of Oncology, Istituto Oncologico Veneto IOV IRCCS, Padua

Alessia Mennitto

Division of Oncology, University Hospital "Maggiore della Carità", Novara

\section{Andrea Sbrana}

Department of Surgical, Medical and Molecular Pathology and Critical Area Medicine, University of Pisa, Pisa

\section{Giandomenico Roviello}

Department of Health Sciences, University of Florence, Firenze

\section{Chiara Casadei}

IRCCS Istituto Romagnolo per lo Studio dei Tumori (IRST) Dino Amadori, Meldola

\section{Pierangela Sepe}

Medical Oncology Department, Fondazione IRCCS Istituto Nazionale dei Tumori, Milano

\section{Sandro Pignata}

Department of Urology and Gynecology, Istituto Nazionale Tumori IRCCS Fondazione "G. Pascale", Napoli

\section{Daniele Santini}

Department of Medical Oncology, Campus Bio-Medico University of Rome, Rome 


\section{Research Article}

Keywords: renal cell carcinoma, immunotherapy, immune-related adverse events

Posted Date: May 11th, 2021

DOl: https://doi.org/10.21203/rs.3.rs-501902/v1

License: (c) (i) This work is licensed under a Creative Commons Attribution 4.0 International License. Read Full License

Version of Record: A version of this preprint was published at Journal of Translational Medicine on August 3rd, 2021. See the published version at https://doi.org/10.1186/s12967-021-03008-9. 


\section{Abstract}

\section{Background}

Severe immune-related Adverse Events (irAEs) develop in 10-27\% of patients treated with anti-Cytotoxic TLymphocyte Antigen (CTLA)-4, in amount $12-20 \%$ of patients treated with anti-programmed cell death (PD)-1 and $15-20 \%$ of patients treated with anti-programmed death-ligand (PD-L)-1(1-3). The aim of our study was to evaluate efficacy and clinical outcome of metastatic renal cell carcinoma (mRCC) patients who stopped Immune Checkpoint Inhibitors (ICls) due to early Grade (G) 3-G4 irAEs.

\section{Methods}

We retrospectively collected data from $204 \mathrm{mRCC}$ patients treated with ICls in 6 Italian referral centers adhering to the Meet-Uro group, between February 2017 and January 2020. To properly weight the results, patients who did not report early G3-G4 toxicities have been included as control group.

Primary endpoint was to evaluate 6 months Progression Free Survival (PFS) after early treatment interruption for Grade (G) 3-4 toxicities compared to the control group. Secondary endpoints were to evaluate Time to treatment failure (TTF) and overall survival (OS) in both groups. All statistical analyses were performed using SPSS software (version 19.00, SPSS, Chicago).

\section{Results}

18/204 (8.8\%) patients had early treatment interruption for serious (G3-G4) irAEs. Early was defined as interruption of $\mathrm{IO}$ after one or two administrations. Immune related nephritis and pancreatitis were the most common irAE that lead to treatment interruption. $6 / 18$ patients received IO-IO combination whereas 12/18 patients antiPD1. In patients who interrupted treatment due to irAE, 12/18 (66.6\%) were free from progression at 6 months from IO interruption, TTF was 1.6 months $(95 \% \mathrm{Cl} 1.6-2.1)$, mPFS was 7.4 months $(95 \% \mathrm{Cl} 3.16-11.6)$ and mOS was 15.5 months (5.1-25.8). In the control group 111/184 (60.3\%) patients were free from progression at 6 months, TTF was 4.6 months $(95 \% \mathrm{Cl} 3.5-5.6)$, mPFS was 4.6 months $(95 \% \mathrm{Cl} 3.5-5.6)$ and mOS was 19.6 months (95\% Cl 15.1-24.0).

In the overall population, mPFS was 5.0 months $(95 \% \mathrm{Cl} 4.0-5.9)$ and mOS was 19.6 months $(95 \% \mathrm{Cl} 15.1$ 24.0).

\section{Conclusions}

ICls seem to maintain efficacy even after early interruption due to severe irAE.

\section{Background}

Immune-Oncology (IO), alone or in combination, changed the paradigm of treatment of metastatic renal cell carcinoma (mRCC) showing an improvement in Overall Survival (OS) and Progression Free Survival 
(PFS) compared to standard of care(4-6). 10 induces tumor death in a different way, compared to Vascular Endothelial Growth Factor Receptors (VEGFR)-Tyrosine Kinase Inibitors (TKI), and, consequently, has different adverse events called immune related Adverse Events (irAEs).

Severe irAEs develop in 10-27\% of patients treated with anti-Cytotoxic T-Lymphocyte Antigen (CTLA)-4, in amount $12-20 \%$ of patients treated with anti-programmed cell death (PD)-1 and $15-20 \%$ of patients treated with anti-programmed death-ligand (PD-L)-1 $(1-3,7)$. In mRCC G3-G4 irAE develop in 1.7-19\% of patients treated with anti-PD1(8) and $1.3-10.4 \%$ of patients treated with anti-PD1 + anti CTLA-4(4).

Fathal irAE have been reported in almost $0.36 \%$ of patients treated with anti PD-1/PDL-1, $1.08 \%$ in those treated with anti-CTLA-4 and $1.23 \%$ of patients treated with combination(9). IrAEs can involve kidney, lung, liver and skin but nervous system and osteoarticular manifestation have been described too(10).

According to recent reports, irAEs correlates with a better outcome. IrAE can arise very early or after long time(11). Long responders' patients have been reported even after a short time to IO exposure.

Therefore, the aim of our study was to retrospectively evaluate the impact of early interruption of IO treatment, due to irAEs, on outcome of mRCC patients.

\section{Methods}

We retrospectively collected data of $\mathrm{mRCC}$ patients treated with $\mathrm{IO}$ in 6 Italian referral centers adhering to the Meet-Uro group, between February 2017 and January 2020.

Inclusion criteria were at least 18 years old at the time of enrollment, histological diagnosis of renal cell carcinoma and radiological diagnosis of metastatic disease.

Patients treated with 10 as single agent or in combination were considered eligible.

Baseline characteristics were collected at the start of IO. Outcome data, including PFS, TTF, OS and toxicities, were collected too. Data included first line treatment, subsequent IO therapy and previous nephrectomy.

The International Metastatic RCC Database Consortium (IMDC) prognostic risk group was computed at the index date based on the presence of six individual risk factors including time from diagnosis to systemic treatment $<1$ year, hemoglobin $<$ lower limit of normal, calcium $>10 \mathrm{mg} / \mathrm{dL}$, platelet $>$ upper limit of normal, neutrophil > upper limit of normal, Performance Status (PS) $<80 \%$ (Karnofsky).

IrAEs were graded according to the National Cancer Institute Common Toxicity Criteria for Adverse Events (CTCAE; version 4.0).

Primary endpoint was to evaluate 6 months PFS after treatment interruption for toxicities. 
Secondary endpoints were to evaluate OS and Time to Treatment Failure (TTF) in patients. TTF was evaluated as the interval from treatment initiation to premature discontinuation due to cancer progression, adverse events patients' choice or death. PFS was defined as the time from treatment start to disease progression or death from any cause.

Early interruption was defined as the interruption of ICls after one or two administration, almost 2 months of treatment.

Patients with no evidence of death were censored at the date of last tumor assessment.

Real-world physician-assessed progression and response was based on clinical criteria or radiographic criteria using Response Evaluation Criteria in Solid Tumors (RECIST) guidelines, with imaging assessments occurring at clinically variable time points.

Baseline demographic and clinical characteristics are described using frequencies and percentages for categorical variables.

Descriptive analysis was made using median values and ranges. All statistical analyses were performed using SPSS software (version 19.00, SPSS, Chicago).

All participating centers received local ethics approval for data collections. The study was conducted in accordance with good clinical practice and the Declaration of Helsinki.

\section{Results}

Data from 204 mRCC patients were retrospectively collected from 6 referral centers. To properly weight the results, patients who did not report early G3-G4 toxicities have been included as control group. Characteristics of patients are described in Table 1. 18/204 (8.8\%) patients had early treatment interruption for serious (G3-G4) irAEs. 
Table 1

Characteristics of patients included in our study. Patients were grouped according to the reason for interruption of ICls due to irAE or progressive disease.

\section{Interruption of 10}

G3-G4 irAE PD

18

Sex

Male

10

98

Female

8

38

Median Age

66.0

60.9

Site of disease

Bone

9

44

Lung

18

73

lymph nodes

12

69

Liver

6

19

Gland

4

28

Synchronous metastatic disease

10

85

Metachronous metastatic disease

8

101

IMDC score

Good

1

74

Intermediate

17

99

Poor

0

13

IO

Single agent

12

178

IO-IO combo

6

8

AID

Y

2

3

$\mathrm{N}$

16

183

irAE (immune-relate Adverse Events); G (grade); PD (progressive disease); IMDC score (International Metastatic RCC Database Consortium); AID (autoimmune disease); ECOG PS (Eastern Cooperative Oncology Group Performance Status); IO (Immune-Oncology); 


\begin{tabular}{|c|c|c|}
\hline & \multicolumn{2}{|c|}{ Interruption of IO } \\
\hline \multicolumn{3}{|l|}{ ECOG PS } \\
\hline 0 & 12 & 96 \\
\hline 1 & 5 & 72 \\
\hline 2 & 1 & 18 \\
\hline \multicolumn{3}{|c|}{ Line of treatment } \\
\hline 1 & 6 & 8 \\
\hline 2 & 7 & 117 \\
\hline 3 & 4 & 49 \\
\hline Further line & 1 & 12 \\
\hline \multicolumn{3}{|c|}{$\begin{array}{l}\text { irAE (immune-relate Adverse Events); G (grade); PD (progressive disease); IMDC score (International } \\
\text { Metastatic RCC Database Consortium); AlD (autoimmune disease); ECOG PS (Eastern Cooperative } \\
\text { Oncology Group Performance Status); IO (Immune-Oncology); }\end{array}$} \\
\hline
\end{tabular}

In the overall population, 190/204 patients received anti-PD1 whereas 14/204 patients were treated with antiCTLA4 + antiPD1 combination. In the early discontinuation group, 6/18 patients received IO-IO combination whereas $12 / 18$ patients antiPD1 $(p<0.0001)$ (Table 3$)$. In the control group, 6 patients developed irAEs after two month of IO treatment. Characteristic of patients who experienced irAEs G3-G4 are described in Table 1 whereas Table 3 reports differences between group of patients who interrupted IO due to irAEs and patients who interrupted treatment due to progression.

In patients who developed early G3-G4 irAEs and then interrupted treatment, 12/18 (66.6\%) patients were free from progression at 6 months from 10 interruption whereas in the control group patients were 69/184 (37.5\%) $p 0.1448$.

Immune related nephritis and pancreatitis were the most common irAEs that lead to early treatment interruption (Table 2). 
Table 2

IrAEs that lead to ICls interruption.

\begin{tabular}{|l|c|}
\hline IrAE & $\mathbf{N}$ \\
\hline Miositis & $\mathbf{1}$ \\
\hline Diabetes mellitus & $\mathbf{1}$ \\
\hline Ipofisitis & $\mathbf{2}$ \\
\hline Nefritis & $\mathbf{3}$ \\
\hline Pneumonitis & $\mathbf{2}$ \\
\hline Injection reaction & $\mathbf{2}$ \\
\hline Colitis & $\mathbf{2}$ \\
\hline Neuro toxicities & $\mathbf{2}$ \\
\hline Pancreatitis & $\mathbf{3}$ \\
\hline Arthritis & $\mathbf{1}$ \\
\hline Ocular toxicities & $\mathbf{1}$ \\
\hline Hemolytic anemia & $\mathbf{1}$ \\
\hline Hepatitis & $\mathbf{2}$ \\
\hline IrAE (immune-related adverse events); ICls (immune checkpoint inhibitors) \\
\hline
\end{tabular}


Table 3

Difference between groups according to G3-G4 irAE interruption.

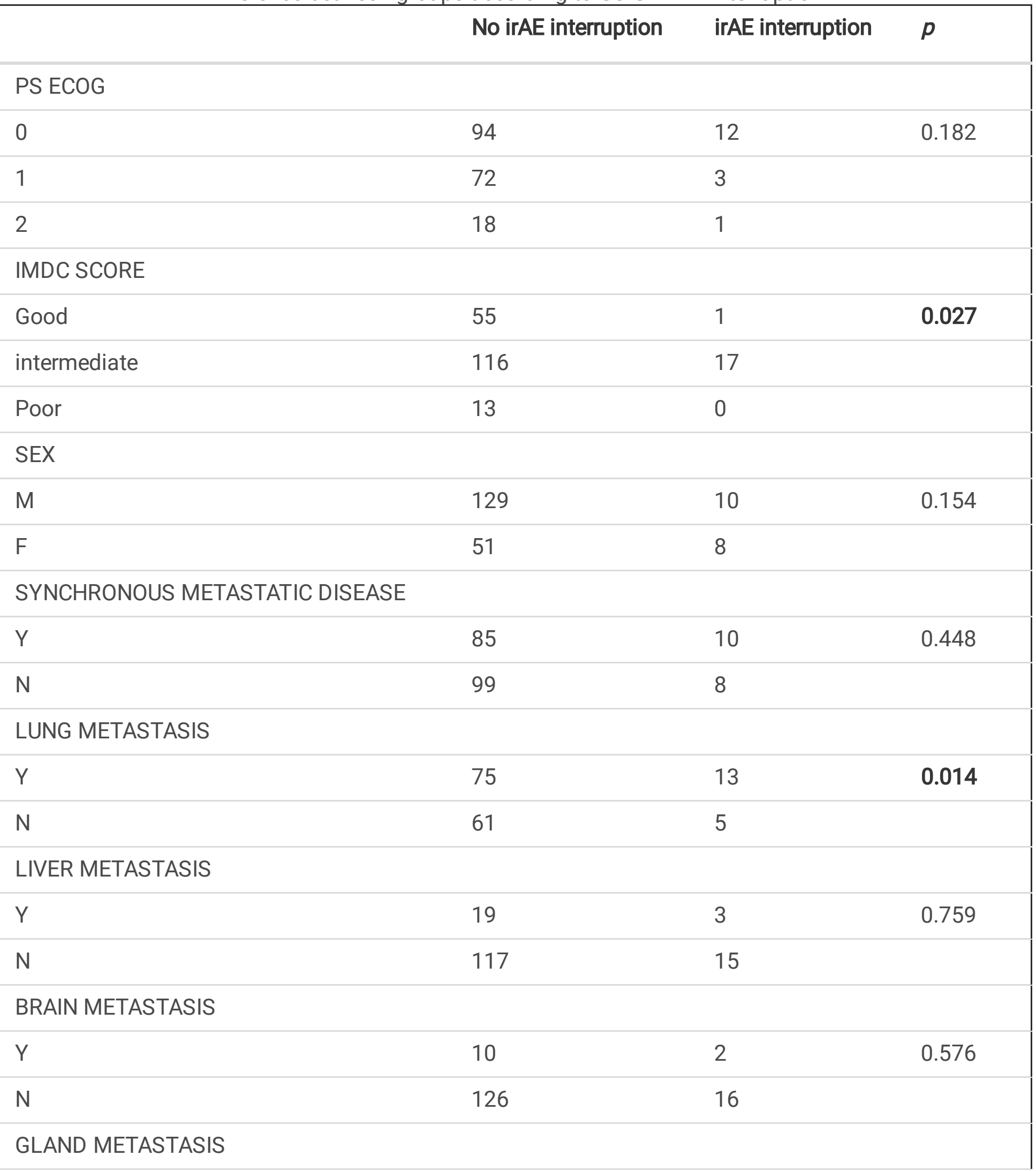

Y (YES); N(No); irAEs (immune-related Adverse Events); IO (Immune-Oncology); IMDC score (International Metastatic RCC Database Consortium); ECOG PS (Eastern Cooperative Oncology Group Performance Status); anti-PD1 (anti programmed death 1); anti-CTLA4 (anti-Cytotoxic T-Lymphocyte Antigen 4); 


\begin{tabular}{|c|c|c|c|}
\hline & No irAE interruption & irAE interruption & $p$ \\
\hline Y & 28 & 2 & 0.340 \\
\hline $\mathrm{N}$ & 108 & 16 & \\
\hline \multicolumn{4}{|l|}{ PERITONEAL } \\
\hline Y & 8 & 0 & 0.367 \\
\hline $\mathrm{N}$ & 176 & 18 & \\
\hline \multicolumn{4}{|l|}{10} \\
\hline Anti-PD1 & 176 & 12 & $<0.0001$ \\
\hline Anti-PD1 + anti CTLA4 & 8 & 6 & \\
\hline \multicolumn{4}{|c|}{$\begin{array}{l}\text { Y (YES); N(No); irAEs (immune-related Adverse Events); IO (Immune-Oncology); IMDC score } \\
\text { (International Metastatic RCC Database Consortium); ECOG PS (Eastern Cooperative Oncology Group } \\
\text { Performance Status); anti-PD1 (anti programmed death 1); anti-CTLA4 (anti-Cytotoxic T-Lymphocyte } \\
\text { Antigen 4); }\end{array}$} \\
\hline
\end{tabular}

In patients who interrupted treatment due to early irAEs, TTF was 1.6 months ( $95 \% \mathrm{Cl} 1.6-2.1)$, mPFS was 7.4 months $(95 \% \mathrm{Cl} 3.16-11.6)$ and mOS was 15.5 months (5.1-25.8). In the control group, 111/184 $(60.3 \%)$ patients were free from progression at 6 months, TTF 4.6 months $(95 \% \mathrm{Cl} 3.5-5.6)$, mPFS was 4.6 months $(95 \% \mathrm{Cl} 3.5-5.6)$ and mOS was 19.6 months $(95 \% \mathrm{Cl} 15.1-24.0)$.

In the overall population, median PFS was 5.0 months $(95 \% \mathrm{Cl} 4.0-5.9)$ and median OS was 19.6 months (95\% Cl 15.1-24.0) whereas TTF was 4.1 months $(95 \% \mathrm{Cl} 3.2-4.9) .125 / 204$ patients were free from progression at 6 months.

\section{Discussion}

IrAEs contribute to mortality and morbidity in $\mathrm{MRCC}$ and represent a relevant issue for healthcare system. Symptomps and signs are often insidiuos and similar to cancer related symptomps so that irAEs rapresent a clinical challenge for phisicians.

Biological biomarkers for irAEs are mostly unknown(12). Baseline circulating Interleukin (IL)-17 was related to G3-G4 colitis(13) whereas Gowen et al reported a different baseline profiling of antibodies in patients who developed irAEs(14).

In clinical practice, pre-existing organ insufficiency is supposed to be associated to higher risk to develop irAEs as well as pre-exiting autoimmune disease (AID)(15). In patients treated with ipilimumab, AID exacerbated in $27 \%$ of cases with $33 \%$ of irAE(16) whereas in patients treated with anti-PD-1, $38 \%$ had a flare of a preexisting AID with $10 \%$ of G3-G4 irAEs and a discontinuation rate of $4 \%(17-19)$. In a larger 
cohort of patients, Tison et al reported AID flare in $47 \%$ of cases and irAEs in $43 \%$ with a discontinuation rate of $21 \%(20)$. Recrudescence of AID and irAEs are usually managed with corticosteroids. The use of immunosuppressive therapy at initiation of $\mathrm{IO}$ is associated to fewer irAEs compared to patients who did not receive corticosteroids.

In our real-world analysis, the incidence of early G3-G4 irAEs was almost $10-15 \%$.

Outcomes here reported, demonstrate the efficacy of 10 even after early interruption due to irAEs. Furthermore, after 6 months from treatment interruption, $66.6 \%$ of patients were free from progression demonstrating long-term benefit from IO. Indeed, G3-4 irAEs seem to be related to efficacy of IO even when treatment is early interrupted due to toxicities(11). Differences in MPFS and mOS between the irAEs group and the control group was not analyzed due to the small sample size and the heterogeneity of patients in groups but the ones who experienced severe irAEs tend to a longer mPFS. Indeed, we reported longer mPFS in severe early irAEs group compared to MPFS reported in the pivotal trials of ICls and other real-world experiences of IO in RCC. Median OS here reported was comparable to previous reports.

The hypothesis underling brilliant response after the onset of irAEs is that an exuberant activation of immune system against our self-tissues becomes an exuberant response against tumor tissue $(11,15)$. Several modification have been seen in the immune system of patients treated with IO, which can explain long-term response(21). In vitro, anti-CTLA4 is associated to an increase expansion and enhance effector function of memory CD8 $+\mathrm{T}$ cell, which can be related to long-term response(22). Instead, it is uncertain if anti-PD1 increases CD8 + effector memory cells in cancer patients. Indeed, as reported in vitro, anti-PD1 enhance cytokine production in human T-cells and, in a T-reg suppression assay, it completely restored CD4 + T-responder cell proliferation and partially restored IFNy production(23).

It is reasonable to suppose that in patients who develop early G3-4 irAEs, ICls promote adapted states of hyper-responsiveness in immune cells, which promote anti-tumor immunity. This subgroup of patients might have a "hyper-immune system" and early G3-G4 irAEs could represent a predictive parameter for persistent adaptations in the immune system, which can persist and display memory-like features. Limits of our study are the small sample size, the retrospective collection of data and the lack of central radiological review. A prospective validation is needed to straighten these findings.

\section{Conclusions}

Pathogenesis and treatment of irAEs represent an interesting research field as it remains under debate. Interruption is often required after irAEs, with consequent doubts about treatment efficacy. Nevertheless, our study, with the limit of a retrospective collection, confirms that in mRCC patients, even after early interruption due to irAEs, IO maintain clinical and radiological efficacy.

\section{Abbreviations}


irAE (immune-relate Adverse Events); G (grade); PD (progressive disease); IMDC (International Metastatic RCC Database Consortium); AID (autoimmune disease); ECOG PS (Eastern Cooperative Oncology Group Performance Status), IO (Immune-Oncology), OS (Overall Survival), PFS (Progression Free Survival), TTF (Time To Treatment Failure), mRCC (metastatic Renal Cell Carcinoma), MSKCC (Memorial Sloane Kattering Cancer Center), Response Evaluation Criteria in Solid Tumors (RECIST), ICls (immunecheckpoint inhibitors), anti-Cytotoxic T-Lymphocyte Antigen (CTLA)-4, anti-programmed cell death (PD)-1, anti-programmed death-ligand (PD-L)-1;

\section{Declarations}

- Ethics approval and consent to participate: All participating centers received local ethics approval for data collections. All patients signed informed consent to participate to the study.

- Consent for publication: Authors received consent for publication.

- Availability of data and material: Authors declare that data and material collected are available after properly request.

- Competing interests: Sandro Pignata received honoraria from MSD AZ ROCHE Pfizer GSK Clovis pharmamar and research funding MSD AZ ROCHE PFIZER. Ugo De Giorgi has served as consultant/advisory board member for Astellas, Bayer, BMS, Ipsen, Janssen, Merck, Pfizer and Sanofi; has received travel support from BMS, Ipsen, Janssen and Pfizer; and has received research funding from AstraZeneca, Roche and Sanofi (Inst). All the other authors have no conflict of interest.

- Funding: None.

- Authors' contributions: All authors contributed equally to the paper.

- Acknowledgements: Authors thank all MeetUro members for their contribution.

\section{References}

1. Powles T, Durán I, van der Heijden MS, Loriot Y, Vogelzang NJ, De Giorgi U, et al. Atezolizumab versus chemotherapy in patients with platinum-treated locally advanced or metastatic urothelial carcinoma (IMvigor211): a multicentre, open-label, phase 3 randomised controlled trial. Lancet (London, England). 2018 Feb;391(10122):748-57.

2. Galsky MD, Arija JÁA, Bamias A, Davis ID, De Santis M, Kikuchi E, et al. Atezolizumab with or without chemotherapy in metastatic urothelial cancer (IMvigor130): a multicentre, randomised, placebocontrolled phase 3 trial. Lancet (London, England). 2020 May;395(10236):1547-57.

3. Haanen JBAG, Carbonnel F, Robert C, Kerr KM, Peters S, Larkin J, et al. Management of toxicities from immunotherapy: ESMO Clinical Practice Guidelines for diagnosis, treatment and follow-up. Ann Oncol [Internet]. 2017;28(Supplement 4):iv119-42. Available from: https://doi.org/10.1093/annonc/mdx225

4. Motzer RJ, Rini BI, McDermott DF, Arén Frontera O, Hammers HJ, Carducci MA, et al. Nivolumab plus ipilimumab versus sunitinib in first-line treatment for advanced renal cell carcinoma: extended 
follow-up of efficacy and safety results from a randomised, controlled, phase 3 trial. Lancet Oncol. 2019 Oct;20(10):1370-85.

5. Motzer RJ, Escudier B, McDermott DF, George S, Hammers HJ, Srinivas S, et al. Nivolumab versus Everolimus in Advanced Renal-Cell Carcinoma. N Engl J Med. 2015;

6. Santini D, Stellato M, De Giorgi U, Pantano F, De Lisi D, Casadei C, et al. Clinical Outcomes of Metastatic Renal Carcinoma Following Disease Progression to Programmed Death (PD)-1 or PD-L1 Inhibitors (IO): A Meet-URO Group Real World Study (Meet-Uro 7). Am J Clin Oncol. 2021 Mar;44(3):121-5.

7. Kumar V, Chaudhary N, Garg M, Floudas CS, Soni P, Chandra AB. Current diagnosis and management of immune related adverse events (irAEs) induced by immune checkpoint inhibitor therapy. Front Pharmacol. 2017;8(FEB).

8. Motzer RJ, Escudier B, George S, Hammers HJ, Srinivas S, Tykodi SS, et al. Nivolumab versus everolimus in patients with advanced renal cell carcinoma: Updated results with long-term follow-up of the randomized, open-label, phase 3 CheckMate 025 trial. Cancer. 2020 Sep;126(18):4156-67.

9. Wang DY, Salem J-E, Cohen J V, Chandra S, Menzer C, Ye F, et al. Fatal Toxic Effects Associated With Immune Checkpoint Inhibitors: A Systematic Review and Meta-analysis. JAMA Oncol [Internet]. 2018 Dec 1;4(12):1721-8. Available from: https://doi.org/10.1001/jamaoncol.2018.3923

10. Postow MA, Sidlow R, Hellmann MD. Immune-Related Adverse Events Associated with Immune Checkpoint Blockade. N Engl J Med [Internet]. 2018 Jan 10;378(2):158-68. Available from: https://doi.org/10.1056/NEJMra1703481

11. Rogado J, Sánchez-Torres JM, Romero-Laorden N, Ballesteros Al, Pacheco-Barcia V, Ramos-Leví A, et al. Immune-related adverse events predict the therapeutic efficacy of anti\&\#x2013;PD-1 antibodies in cancer patients. Eur J Cancer [Internet]. 2019 Mar 1;109:21-7. Available from: https://doi.org/10.1016/j.ejca.2018.10.014

12. Raimondi A, Sepe P, Zattarin E, Mennitto A, Stellato M, Claps M, et al. Predictive Biomarkers of Response to Immunotherapy in Metastatic Renal Cell Cancer. Front Oncol. 2020;10:1644.

13. Tarhini AA, Zahoor H, Lin Y, Malhotra U, Sander C, Butterfield LH, et al. Baseline circulating IL-17 predicts toxicity while TGF- $\beta 1$ and IL-10 are prognostic of relapse in ipilimumab neoadjuvant therapy of melanoma. J Immunother Cancer [Internet]. 2015 Dec 1;3(1):39. Available from: http://jitc.bmj.com/content/3/1/39.abstract

14. Nishino M, Sholl LM, Hatabu H, Ramaiya NH, Hodi FS. Anti-PD-1-Related Pneumonitis during Cancer Immunotherapy. N Engl J Med. 2015 Jul;373(3):288-90.

15. Abdel-Wahab N, Shah M, Lopez-Olivo MA, Suarez-Almazor ME. Use of Immune Checkpoint Inhibitors in the Treatment of Patients With Cancer and Preexisting Autoimmune Disease: A Systematic Review. Ann Intern Med. 2018 Jan;168(2):121-30.

16. Johnson DB, Sullivan RJ, Ott PA, Carlino MS, Khushalani NI, Ye F, et al. Ipilimumab Therapy in Patients With Advanced Melanoma and Preexisting Autoimmune Disorders. JAMA Oncol. 2016 Feb;2(2):234-40. 
17. Brahmer JR, Drake CG, Wollner I, Powderly JD, Picus J, Sharfman WH, et al. Phase I study of singleagent anti-programmed death-1 (MDX-1106) in refractory solid tumors: Safety, clinical activity, pharmacodynamics, and immunologic correlates. J Clin Oncol. 2010;28(19):3167-75.

18. Menzies AM, Johnson DB, Ramanujam S, Atkinson VG, Wong ANM, Park JJ, et al. Anti-PD-1 therapy in patients with advanced melanoma and preexisting autoimmune disorders or major toxicity with ipilimumab. Ann Oncol Off J Eur Soc Med Oncol. 2017 Feb;28(2):368-76.

19. Gutzmer R, Koop A, Meier F, Hassel JC, Terheyden P, Zimmer L, et al. Programmed cell death protein-1 (PD-1) inhibitor therapy in patients with advanced melanoma and preexisting autoimmunity or ipilimumab-triggered autoimmunity. Eur J Cancer. 2017 Apr;75:24-32.

20. Tison A, Quéré G, Misery L, Funck-Brentano E, Danlos F-X, Routier E, et al. Safety and Efficacy of Immune Checkpoint Inhibitors in Patients With Cancer and Preexisting Autoimmune Disease: A Nationwide, Multicenter Cohort Study. Arthritis Rheumatol (Hoboken, NJ). 2019 Dec;71(12):2100-11.

21. Teraoka S, Fujimoto D, Morimoto $T$, Kawachi $H$, Ito M, Sato $Y$, et al. Early Immune-Related Adverse Events and Association with Outcome in Advanced Non-Small Cell Lung Cancer Patients Treated with Nivolumab: A Prospective Cohort Study. J Thorac Oncol Off Publ Int Assoc Study Lung Cancer. 2017 Dec;12(12):1798-805.

22. van der Vlist M, Kuball J, Radstake TRD, Meyaard L. Immune checkpoints and rheumatic diseases: what can cancer immunotherapy teach us? Nat Rev Rheumatol [Internet]. 2016;12(10):593-604. Available from: https://doi.org/10.1038/nrrheum.2016.131

23. Pedicord VA, Montalvo W, Leiner IM, Allison JP. Single dose of anti-CTLA-4 enhances CD8\&lt;sup\&gt; \&lt;/sup\&gt; T-cell memory formation, function, and maintenance. Proc Natl Acad Sci [Internet]. 2011 Jan 4;108(1):266 LP - 271. Available from: http://www.pnas.org/content/108/1/266.abstract 